Therefore when $\bar{C}$ is any curve in $F^{\prime}$ and $F^{\prime \prime}$ joining the points 1 and 3 and $C$ is the corresponding curve satisfying conditions I, II, III, IV', the preceding equations give the relation

$$
J^{*}\left(\bar{C}_{12^{\prime}}\right)+j^{*}\left(\bar{C}_{2^{\prime} 3}\right)=J^{*}\left(C_{12}\right)+j^{*}\left(C_{23}\right)=J\left(C_{12}\right)+j\left(C_{23}\right) .
$$

This equation may also be written in the form

$$
\begin{aligned}
J\left(\bar{C}_{12^{\prime}}\right)+j\left(\bar{C}_{2^{\prime} 3}\right)- & {\left[J\left(C_{12}\right)+j\left(C_{23}\right)\right] } \\
& =J\left(\bar{C}_{12^{\prime}}\right)-J^{*}\left(\bar{C}_{12}\right)+j\left(\bar{C}_{2^{\prime} 3}\right)-j^{*}\left(\bar{C}_{2^{\prime} 3}\right) \\
& =\int_{\bar{C}_{1^{\prime}}} E d t+\int_{\bar{C}_{2^{\prime} 3}} e d t,
\end{aligned}
$$

where $E$ and $e$ are the extended Weierstrass $E$-functions. On account of II these functions are nowhere negative and therefore

$$
J\left(\bar{C}_{12^{\prime}}\right)+j\left(\bar{C}_{2^{\prime} 3}\right)>J\left(C_{12}\right)+j\left(C_{23}\right) .
$$

Hence the conclusion: Under the hypothesis imposed the curve $C_{123}$ actually minimizes the sum of the integrals $J$ and $j$ if it satisfies the conditions I, II, III, IV'.

Sheffield Scientific School,

YALE UNIVERSITY.

\title{
THE DEGREE OF A CARTESIAN MULTIPLIER.
}

BY PROFESSOR D. R. CURTISS.

(Read before the Chicago Section of the American Mathematical Society, April 8, 1910, and April 5, 1912.)

1. A large part of Laguerre's numerous and important contributions to the theory of algebraic equations* is based on Descartes' rule of signs, and especially on its application to infinite series. One of the most fertile ideas developed is that an upper limit for the number of real roots of a polynomial with real coefficients, $f(x)$, in an interval $[0, a]$ results from the application of the rule of signs to a product $f_{2}(x)=f_{1}(x) f(x)$ developed in a power series which converges for $|x|<a$, but

* See in particular the memoir, "Sur la théorie des équations numériques," Oeuvres, pp. $3-47$. 
diverges for $x=a$. Thus integral functions $f_{1}(x)$ exist for which this method gives, in general, a better approximation for the number of positive real roots of $f(x)$ than could be obtained by the direct use of the rule of signs on $f(x)$ itself. In particular Laguerre has shown (loc. cit.) that if the product $e^{z x} f(x)$ is developed according to ascending powers of $x$, the number of variations of sign in the sequence of the coefficients will be exactly equal to the number of positive real roots of $f(x)$, provided $z$ is a sufficiently large positive number. Recently it has been proved by Fekete and Pólya* that the rule of signs applied to the power series for $f(x) /(1-x)^{n}$ gives the exact number of real roots of $f(x)$ in the interval $[0,1]$ if $n$ is sufficiently large, a result easily modified so as to apply to the interval $[0, \infty]$; and in the same paper it is announced that the multiplier $(1+x)^{n}$, with a similar restriction on $n$, may also be used to obtain the number of positive real roots.

A question of particular interest is the existence and character for a given polynomial with real coefficients, $f(x)$, of what I have elsewhere $\dagger$ styled Cartesian multipliers, i. e., polynomials $f_{1}(x)$ such that the rule of signs applied to the product $f_{2}(x)=f_{1}(x) f(x)$ gives exactly the number of positive real roots of $f(x)$. A discussion of such multipliers for polynomials having no positive roots has been given by E. Meissner, $\ddagger$ and in the general case by the present writer in the paper above cited, where their existence is made a corollary of Laguerre's theorem concerning the product $e^{z x} f(x)$. Their existence, of course, also follows from the results obtained by Fekete and Pólya.

In the present paper I shall give a proof of the existence of Cartesian multipliers which is in some ways simpler than any yet given.§ That the construction of a multiplier by the method indicated would require a knowledge of the roots seems an objection, but this is met by a theorem of my Annalen paper above referred to, which states that if there is a Cartesian multiplier of degree $r$, there is always at least one of,

* "Über ein Problem von Laguerre," Palermo Rendiconti, vol. 34 (1912), p. 89.

$\dagger$ " $\mathrm{An}$ extension of Descartes' rule of signs," Math. Annalen, vol. 73 (1912)..p. 424 .

¥ "Über positive Darstellungen von Polynomen," Math. Annalen, vol. $70(1911)$, p. 223.

$\$$ This proof possibly antedates the others, having been announced in April, 1910, as noted above. 
that degree for which the product function $f_{2}(x)$ has no more than $n+1$ non-vanishing coefficients, where $n$ is the degree of $f(x)$. The number of product functions of degree $r+n$ with $r$ terms lacking is evidently finite; if we pick out those functions of this set whose coefficients present the minimum number of variations of sign, and divide each by $f(x)$, we obtain Cartesian multipliers whose coefficients are rational in the coefficients of $f(x)$. From this point of view it is important to obtain as low a value for $r$ as possible, and in a form not requiring a knowledge of the roots of $f(x)$. Since $x^{k} f_{1}(x)$ is for all positive integral values of $k$ a Cartesian multiplier of $f(x)$ if $f_{1}(x)$ has this property, it is obvious that there exist Cartesian multipliers of all degrees greater than the minimum $r$. Although the evaluation for the degree of a Cartesian multiplier obtained in the following pages is far too large, in general, for practical applications, it has at least the interest of a first attempt at an explicit formula.

2. We shall consider first the case where $f(x)$ is a quadratic with imaginary roots. For a very ingenious geometric method the reader should consult the paper of Meissner above cited.

The case of interest is, of course, where $f(x)$ has the form $x^{2}-a x+b\left(a^{2}<4 b\right), a$ and $b$ being positive real numbers, this being the only form for which Descartes' rule is inexact. The problem is to assign a multiplier $f_{1}(x)$ such that the product $f(x) f_{1}(x)$ will have no negative coefficients.

An especially simple solution is the following: If we multiply $x^{2}-a x+b$ by $x^{2}+a x+b$, the result will be of the form $x^{4}-a_{1} x^{2}+b^{2}$; if $a_{1}$ is negative, we stop here, otherwise we multiply the last expression by $x^{4}+a_{1} x^{2}+b^{2}$, and keep up the process thus indicated. When this has been carried sufficiently far we shall obtain a product of the form $x^{2^{k+1}}-$ $a_{k} x^{2^{k}}+b^{2^{k}}$, where $a_{k}$ is negative or zero. The truth of this is evident if the roots of $f(x)$ are $\rho e^{i \phi}, \rho e^{-i \phi}$, so that $a=2 \rho \cos \phi$ and $b=\rho^{2}$; it then follows at once that $a_{1}=2 \rho^{2} \cos 2 \phi$, and $a_{k}=2{\rho^{2}}^{k} \cos 2^{k} \phi$. Since $a$ is positive, $\phi$ can be taken as an angle of the first quadrant, and $k$ may be chosen as the least positive integer for which $2^{k} \phi \geqq \pi / 2$.

The value $2^{k+1}-2$ thus obtained as the degree of a Cartesian multiplier for a quadratic of the above form is not, in general, the minimum one. This has been proved by Meissner, in the paper cited, to be the least positive integer $r$ for which $(r+2) \phi \geqq \pi$. The same result has been obtained by the 
author (loc. cit.) as a corollary of the theorem that there is a Cartesian multiplier of minimum degree such that the product function is of form $x^{r+2}+A x^{s}+B$. The coefficients $A$ and $B$ are easily determined from the fact that the roots of $f(x)$ must be roots of the product; $s$ may be any positive integer less than $r+2$. The corresponding Cartesian multipliers are easily computed.

Though a solution of our problem in this case has been thus obtained, the interest attaching to Laguerre's multiplier $e^{z x}$ will perhaps warrant a brief digression to examine the Cartesian multiplier formed by taking $m+1$ terms of its development in powers of $x$. If the product is arranged in the form

$$
c_{0}+c_{1} x+\cdots+c_{m+2} x^{m+2},
$$

the coefficients are given by the formulas

$$
\begin{aligned}
c_{k} & =\frac{z^{k-2}}{k !}\left(\rho^{2} z^{2}-2 \rho z k \cos \phi+k(k-1)\right) \quad(k \leqq m), \\
c_{m+1} & =\frac{z^{m-1}}{m !}(-2 \rho z \cos \phi+m), \\
c_{m+2} & =\frac{z^{m}}{m !} .
\end{aligned}
$$

We can make $c_{k}$ positive or zero for all values of $k \leqq m$, no matter how large $m$ is taken, if

$$
z=\frac{1}{2 \rho(1-\cos \phi)},
$$

and $c_{m+1}$ will then be positive if

$$
m \geqq \frac{\cos \phi}{1-\cos \phi} .
$$

If we compare this degree $m$ with $r$ in the preceding paragraph, we see that $m$ is never less than $r$, and is much larger for small values of $\phi$.

3. We shall now form a multiplier for any polynomial $f(x)$ of degree $n$ by a method which depends upon a knowledge of the roots, leaving for the following section the problem of obtaining an upper limit $M$ for the degree of this multiplier in an explicit expression in terms of the coefficients of $f(x)$. 
According to $\S 1$ we are then assured of the existence of Cartesian multipliers of all degrees $\geqq M$ whose coefficients are rational in terms of those of $f(x)$.

Let us write our polynomial of degree $n$ in the form

$$
f(x)=p_{2 n_{1}}(x) q_{n_{2}}(x) s_{n_{3}^{\prime}}^{\mathrm{l}}(x),
$$

where each of the factors $p_{2 n_{1}}, q_{n_{2}}, s_{n_{8}}$, is a polynomial of the degree indicated by its subscript, the first having imaginary roots only, the second only zero or negative roots, and the last only positive roots. If $n_{1}=0$ the rule of signs for $f(x)$ is exact, hence the case to be considered is that where $n_{1}>0$.

We have just proved that each quadratic factor of $p_{2 n_{1}}$ has a multiplier such that the product has all its terms positive. The product $P(x)$ of these multipliers will then be a Cartesian multiplier for $p_{2 n_{1}}(x) q_{n_{2}}(x)$, since the coefficients of $q_{n_{2}}(x)$ are all of like sign. Let us designate the degree of the product $P(x) p_{2 n_{1}}(x) q_{n_{2}}(x)$ by $N$. Since we are considering the case where $n_{1}>0$, we must have $N \geqq 2$.

The polynomial $s_{n_{3}}(x)$ has the factored form

$$
s_{n_{3}}(x)=k \prod_{i=1}^{i=n_{8}}\left(x-\alpha_{i}\right),
$$

where $k$ is a real constant, and the numbers $\alpha_{i}$ are all real and positive. If this is multiplied by

$S(x)=\prod_{i=1}^{i=n_{3}}\left(x^{N-1}+x^{N-2} \alpha_{i}+\cdots+x^{N-h} \alpha_{i}{ }^{h-1}+\cdots+\alpha_{i}{ }^{N-1}\right)$,

where $N$ has the value indicated above, we shall have

$$
S(x) s_{n_{3}}(x)=k \prod_{i=1}^{i=n_{3}}\left(x^{N}-\alpha_{i}{ }^{N}\right)=k \sum_{j=0}^{j=n_{8}}(-1)^{j} \lambda_{j} x^{\left(n_{3}-j\right) N},
$$

where every $\lambda_{j}$ is positive. It is now evident, if $S(x) s_{n_{3}}(x)$ and $P(x) p_{2 n_{1}}(x) q_{n_{2}}(x)$ are arranged as polynomials in descending powers of $x$, that their product will present $n_{3}+1$ sequences of terms such that all coefficients of the same sequence will have the same sign, which will be opposite to that of the coefficients in the succeeding sequence. The product will thus present $n_{3}$ variations of sign, where $n_{3}$ is the number of positive real roots of $f(x)$. But

$$
P(x) p_{2 n_{1}}(x) q_{n_{2}}(x) \cdot S(x) s_{n_{3}}(x)=P(x) S(x) f(x),
$$

so that $P(x) S(x)$ is a Cartesian multiplier of $f(x)$. 
4. It remains to obtain an upper limit for the degree of the multiplier $P(x) S(x)$ described in $\S 3$, and to put this in a form depending explicitly on the coefficients of $f(x)$.

The polynomial $P(x)$ was obtained as the product of Cartesian multipliers for each of the quadratic factors of $p_{2 n_{1}}(x)$. Hence if $m_{1}$ is not less than the degree of any of these multipliers, the degree of $P(x)$ will be at most $m_{1} n_{1}$. From its definition, the number $N$ verifies the equation

$$
N=m_{1} n_{1}+2 n_{1}+n_{2},
$$

if $m_{1} n_{1}$ is the degree of $P(x)$. Since

$$
n=2 n_{1}+n_{2}+n_{3}
$$

the above may be written

$$
N=m_{1} n_{1}+n-n_{3} .
$$

Hence, the degree of $S(x)$ being $n_{3}(N-1)$, the degree of $P(x) S(x)$ is at most equal to

$$
m=m_{1} n_{1}+n_{3}(N-1)=m_{1} n_{1}\left(1+n_{3}\right)+n_{3}\left(n-n_{3}-1\right) .
$$

But

so that

$$
2 n_{1} \leqq n-n_{3}
$$

$$
m \leqq m_{1} \frac{n-n_{3}}{2}\left(1+n_{3}\right)+n_{3}\left(n-n_{3}-1\right) .
$$

This expression is a quadratic in $n_{3}$ whose maximum value, reached when $n_{3}=(n-1) / 2$, is easily obtained. In case this is an integer, it will serve for $M$, or, more generally,

$$
M=E\left[\left(\frac{m_{1}}{2}+1\right)\left(\frac{n-1}{4}\right)^{2}+\frac{m_{1}}{2} n\right],
$$

where the symbol $E(a)$ denotes the largest integer not greater than the real number $a$.

This number $M$ will be the degree of a Cartesian multiplier in all cases. To be sure, it was obtained under the hypothesis $n_{1}>0$, but as before observed, if $n_{1}=0$ the rule of signs is exact for $f(x)$ itself, so that $x^{M}$, for instance, will be a multiplier of degree $M$.

We now proceed to express the number $m_{1}$ in the above 
formula in terms of the coefficients of $f(x)$. This number, it will be recalled, is a positive integer large enough to be the degree of a Cartesian multiplier for any quadratic factor of $f(x)$ corresponding to a pair of conjugate imaginary roots.

We have seen in $\S 2$ that a quadratic

$$
x^{2}-2 \rho \cos \phi \cdot x+\rho^{2}
$$

has a multiplier of degree $r$, where $r$ is the least positive integer such that

$$
(r+2) \phi \geqq \pi,
$$

and there will be multipliers of any degree greater than that given by this formula. It follows that if $\psi$ is a positive angle less than all the positive arguments of the imaginary roots of $f(x)$, we can take for $m_{1}$ the least positive integer verifying the inequality

$$
\left(m_{1}+2\right) \psi \geqq \pi .
$$

A simple method for obtaining an evaluation for $\psi$ in terms of the coefficients of $f(x)$ is suggested by Cauchy's treatment of the problem of determining a minimum absolute value for the differences of real roots of a polynomial.*

Since the removing of multiple roots from $f(x)$ involves only rational processes, we will here suppose that they are absent. Let the roots of $f(x)$, all distinct, be designated by $x_{1}, x_{2}, \cdots$, $x_{n}$, and the discriminant of $f(x)$ by $D$. Then, by a well-known formula $\dagger$

$$
\begin{gathered}
D=(-1)^{\frac{n(n-1)}{2}} a_{0}{ }^{2 n-2} \prod_{\lambda, \mu}\left(x_{\lambda}-x_{\mu}\right)^{2} \\
(\lambda=1,2, \cdots, n-1 ; \mu=\lambda+1, \lambda+2, \cdots, n) .
\end{gathered}
$$

If the minimum positive argument of imaginary roots is $\phi_{1}$, the corresponding pair of roots being $\rho_{1} e^{ \pm i \phi_{1}}$, and if $\rho$ denotes a superior limit for the moduli of roots of $f(x)$, $\ddagger$ we have, since $\left|x_{\lambda}-x_{\mu}\right| \leqq 2 \rho$,

or

$$
\begin{aligned}
|D| & \leqq\left|a_{0}\right|^{2 n-2}\left(2 \rho_{1} \sin \phi_{1}\right)^{2}(2 \rho)^{n(n-1)-2} \\
& \leqq\left|a_{0}\right|^{2 n-2}(2 \rho)^{n(n-1)}\left(\sin \phi_{1}\right)^{2}
\end{aligned}
$$

* Netto, Vorlesungen über Algebra, vol. 1, p. 272.

$\dagger$ Netto, 1. c., p. 177.

$¥$ Such a limit is, for example, given by the formula $\rho=1+r / r_{0}$, where $r_{0}=\left|a_{0}\right|$, and $r$ is the greatest of the numbers $\left|a_{0}\right|,\left|a_{1}\right|, \cdots,\left|a_{n}\right|$. 


$$
\sin \phi_{1} \geqq \frac{|D|^{\frac{1}{2}}}{\left|a_{0}\right|^{n-1}(2 \rho)^{n(n-1) / 2}} .
$$

Solved for $\phi_{1}$, this gives

$$
\phi_{1} \geqq \sin ^{-1} \frac{|D|^{\frac{1}{2}}}{\left|a_{0}\right|^{n-1}(2 \rho)^{n(n-1) / 2}} .
$$

Since $\phi_{1} \geqq \sin \phi_{1}$, (4) may be replaced by

$$
\phi_{1} \geqq \frac{|D|^{\frac{1}{2}}}{\left|a_{0}\right|^{n-1}(2 \rho)^{n(n-1) / 2}} .
$$

If the coefficients of $f(x)$ are integers, $D$ is a whole number, and $|D| \geqq 1$, so that (4) and (5) can in this case be replaced by the stronger but simpler inequality

$$
\phi_{1} \geqq \frac{1}{\left|a_{0}\right|^{n-1}(2 \rho)^{n(n-1) / 2}} .
$$

We may take for $\psi$ the right-hand side of (4) or (5) or, in the case indicated, of (6).

The problem of obtaining the degree of a Cartesian multiplier is thus solved by formulas (2) and (3), $\psi$ being taken equal to the right-hand member of either (4), (5), or (6).

It must be confessed that the evaluation thus obtained is absurdly high, even in simple cases. Thus, to take an example somewhat at random, if $f(x)=x^{4}+x^{3}-2 x^{2}+2 x+3$, the least value of $m_{1}$ as given by (3) and (4) is not far from 8,500 , and (2) would give $M$ as greater than 19,000. I have shown in my Annalen paper that this polynomial has a Cartesian multiplier of degree 2 . It is not likely that the methods here given, even if improved, will have much value for numerical computation. There remains, however, the interest attaching to a formal solution of the problem in hand.

NORTHWESTERN UNIVERSITY. 